\title{
Progress in studies on bulk metallic glasses in China
}

Bulk metallic glasses (BMGs) are a new class of metallic materials possessing a unique microstructure without long-range order, in contrast to crystalline solids which have periodic arrangements of atoms in space. Since the successful production of bulk metallic glasses by copper mold casting around the end of the 1980s, great enthusiasm in the study on this new class of materials has been stimulated, and many bulk metallic glasses have been developed [1]. They exhibit extremely high strength, good magnetic properties, excellent superplasticity in the supercooled liquid region, high corrosion resistance and many other scientifically and technologically attractive properties which have not been obtained for conventional crystalline alloys [1]. This means that BMGs have potential applications in various fields and are also important for a broader understanding of condensed matter.

Over the past decade, extensive studies on BMGs in China have been conducted and received substantial support from the Ministry of Science and Technology and National Natural Science Foundation of China. Scientists in China have made remarkable progress in studies on the synthesis, development, microstructure, properties and applications of bulk metallic glasses, and their work in the field of metallic glasses has attracted great attention internationally.

At the ceremony for the 2010 National Science and Technology Award, held on January 14, 2011, the project 'Study on the formation mechanism of metallic glasses and development of novel rare earth metal-based bulk metallic glasses', which was completed by Professors Weihua WANG, Mingxiang PAN, Deqian ZHAO and Haiyang BAI from The Institute of Physics, Chinese Academy of Sciences (CAS), received second prize in the National Natural Science Awards. The main discoveries of this project were (1) the elastic modulus criteria for controlling the properties and formation of metallic glasses by tuning the elastic modulus, (2) development of metallic glassy materials with combined properties of metals and plastics metallic plastics, and (3) synthesizing novel rare earth metal-based metal glasses according to the elastic modulus criteria. The research work not only leads to more profound understanding of the formation mechanisms of amorphous materials, but also greatly contributes to formation and development of metallic glasses, especially to the syntheses of rare earth metal based BMGs with potential applications. It was the first time for scientists in the field of BMGs to gain this very important award, which will stimulate more active research on BMGs in China.

Many other experimental and theoretical discoveries in the field of bulk metallic glasses have also been made in China in recent years. With the contribution and efforts of colleagues in China, two special issues of Science China Physics, Mechanics \& Astronomy on BMGs have been published (in March 2010 and April 2008 [2,3]). These special issues constitute a valuable collection of recent Chinese achievements in this exciting materials field, including the structure, composition design, formation, preparation, and mechanical, physical and chemical properties of bulk metallic glasses. The special issues provide researchers with an update on recent progress in understanding and applications of BMGs; their publication is expected to promote further advancement of BMGs in China.

Great advances in the fundamentals as well as the applications of BMGs have been made; meanwhile there remain many challenges for this new class of materials. It is believed that researchers in China will achieve further important and highly influential accomplishments in this exciting materials field, enabling further progress in the study on BMGs.

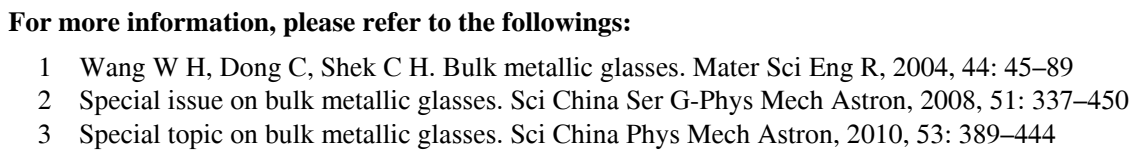

Open Access This article is distributed under the terms of the Creative Commons Attribution License which permits any use, distribution, and reproduction in any medium, provided the original author(s) and source are credited. 